POS PROCEEDINGS

\title{
Rare and forbidden decay of charm mesons at CLEO-C and BES-III
}

\author{
Hai-Bo Li ${ }^{* \dagger}$ \\ Author Institute of High Energy Physics, P.O.Box 918, Beijing 100049, China \\ E-mail: lihbeihep.ac.cn
}

\begin{abstract}
This paper presents the results of searches for the flavor-changing neutral current, lepton-numberviolating and lepton-flavor-violating processes in the charm mesons decays. Recent results from charged $D$ mesons decays to final states with dielectrons from CLEO-c are reported. The prospects of the searches for the rare charm decays at BES-III are also discussed.
\end{abstract}

KAON International Conference

May 21-25 2007

Laboratori Nazionali di Frascati dell'INFN, Rome, Italy

\footnotetext{
*Speaker.

${ }^{\dagger}$ For BES-III and CLEO-c Collaborations.
} 


\section{Introduction}

Searches for rare-decay processes have played an important role in the development of the Standard Model (SM). The absence of flavor-changing neutral current (FCNC) in $K$ decays implied the existence of charm quark, and the observations of $B^{0}-\bar{B}^{0}$ and $B_{s}-\bar{B}_{s}$ mixings (FCNC processes) signaled the very large top-quark mass. The study of the FCNC has been focused on rare decays involving transitions such as $s \rightarrow d l^{+} l^{-}, s \rightarrow d v \bar{v}, b \rightarrow s \gamma$, and $b \rightarrow l^{+} l^{-}$.

In contrast with the $K$ and $B$ FCNC processes, the $D$ meson FCNC transitions are mediated by light down-quark sector, which implies an efficient Glashow-Iliopoulos-Maiani (IM) cancellation. In this case, $D^{0}$ mixing, as well as FCNC decays are expected to be very small in the SM short-distance contributions. Many extensions of the SM may enhance the mixing rate and FCNC processes in the short-distance of the $D$ system by orders of magnitudes. Unfortunately, the new physics contributions are diluted by large long-distance contributions which are likely to dominate over the SM short-distance effects. For example, the charm radiative decays $D \rightarrow h \gamma$, where $h$ denotes a light hadron final state [1], are completely dominated by hadronic uncertainty. However, semileptonic decays such as $c \rightarrow u l^{+} l^{-}$may be used to constrain the new physics since one can look at the new physics contributions to the whole kinematics to which the new physics may contribute in the region away from the resonance-dominated (long-distance dominated) region [2, 3]. Although the long-distance effects dominate the $D^{0}$ mixing rate, the recent experimental bounds can still be used to constrain the new physics space. Purely leptonic flavor-violating (LFV) modes such as $D^{0} \rightarrow \mu^{ \pm} e^{\mp}$ and $D \rightarrow h \mu^{ \pm} e^{\mp}$, as well as lepton-number violated (LNV) modes $D^{+} \rightarrow h^{-} e^{+} e^{+}$ , are completely not allowed in the SM, they are "smoking gun" for new physics searches. In this paper, we report the recent measurements of rare and forbidden charm decays from CLEO-c, and discuss the prospect of these decays at the BES-III experiment. A comparison between $B$ factories and $\tau$-charm factories are also presented.

\section{CLEO-c and BES-III for charm flavor physics}

The Cornell Electron Storage Ring (CESR) had been upgraded to CESR-c with the installation of 12 wiggler magnets to increase damping at low energy. The CLEO-c detector is a minimal modification of the well understood CLEO-III detector. It is the first modern detector to operate at charm threshold. The CLEO-c has accumulated a total of $281 \mathrm{pb}^{-1}$ at $\psi(3770)$ peak (1.8 million $D \bar{D}$ pairs) and $200 \mathrm{pb}^{-1}$ at $\sqrt{s}=4170 \mathrm{MeV}$ for $D_{s}$ physics. CLEO-c expects to take data until April 2008 and will approximately triple each data set by that time [4].

The BES-III detector is designed for the $e^{+} e^{-}$collider running at the $\tau$-Charm energy region, called BEPCII, which is currently under construction at IHEP, Beijing, P.R. China [5]. The accelerator has two storage rings with a circumference of $224 \mathrm{~m}$, one for electron and one for positron, each one with 93 bunches spaced by $8 \mathrm{~ns}$. The total current of the beam is $0.93 \mathrm{amp}$, and the crossing angle of two beams is designed to be $22 \mathrm{mrad}$. The peak luminosity is expected to be $10^{33} \mathrm{~cm}^{-2} \mathrm{~s}^{-1}$ at the beam energy of $1.89 \mathrm{GeV}$, the bunch length is estimated to be $1.5 \mathrm{~cm}$ and the energy spread will be $5.16 \times 10^{-4}$. At this moment, the LINAC has been installed and successfully tested, with all specifications satisfied. The storage rings have been installed, and will be commissioned for synchrotron radiation run by the end of the year. 
The BES-III detector consists of a He-based small cell drift chamber, Time-Of-Flight (TOF) counters for PID, a CsI(Tl) crystal calorimeter, a solenoidal super-conducting magnet with a field of 1 Tesla and the return yoke interleaved with Resistive Plate Chambers (RPC) counters acting as muon chamber. The construction is expected to be completed in the middle of 2007. Photon energy resolution is $\Delta E / E=2.5 \%$ at $E_{\gamma}=1.0 \mathrm{GeV}$. The momentum resolution is $\sigma_{p} / p=0.5 \%$ at $p=1.0 \mathrm{GeV} / c$, and the $d E / d x$ resolution for hadron tracks is about $6 \%$. The time resolution of TOF is about $100 \mathrm{ps}$, combining energy loss $(d E / d x)$ measurement in the drift chamber gives 10 $\sigma \mathrm{K} / \pi$ resolution across the typical kinematic range.

Table 1: $\tau$-Charm productions at BEPC-II in one year's running $\left(10^{7} s\right)$.

\begin{tabular}{llll}
\hline Data Sample & $\begin{array}{l}\text { Central-of-Mass energy } \\
(\mathrm{MeV})\end{array}$ & $\begin{array}{l}\text { Luminosity } \\
\left(10^{33} \mathrm{~cm}^{-2} \mathrm{~s}^{-1}\right)\end{array}$ & $\begin{array}{l}\text { \#Events } \\
\text { per year }\end{array}$ \\
\hline$J / \psi$ & 3097 & 0.6 & $10 \times 10^{9}$ \\
$\tau^{+} \tau^{-}$ & 3670 & 1.0 & $12 \times 10^{6}$ \\
$\psi(2 S)$ & 3686 & 1.0 & $3.0 \times 10^{9}$ \\
$D^{0} \bar{D}^{0}$ & 3770 & 1.0 & $18 \times 10^{6}$ \\
$D^{+} D^{-}$ & 3770 & 1.0 & $14 \times 10^{6}$ \\
$D_{S}^{+} D_{S}^{-}$ & 4030 & 0.6 & $1.0 \times 10^{6}$ \\
$D_{S}^{+} D_{S}^{-}$ & 4170 & 0.6 & $2.0 \times 10^{6}$ \\
\hline
\end{tabular}

The BES-III can accumulate $10 \times 10^{9} \mathrm{~J} / \psi, 3 \times 10^{9} \psi(2 S), 30$ million $D \bar{D}$ or 2 million $D_{S} \bar{D}_{S^{-}}$ pairs per running year as listed in Table 1, respectively, when it is turned to run at resonances in 2008 [6]. Coupled with what is available at CLEO-c, the BES-III will make it possible for the first time to study in detail the light hadron spectroscopy in the decays of charmonium states and the charmed mesons. In addition, about 30 million $D \bar{D}$ pairs will be collected at BES-III in one year at $\psi(3770)$ peak. With modern techniques and unprecedented high statistical data sample, Searching for rare D, Charmonium and tau decays will be possible, such as LFV and LNV decays, invisible decays.

\section{Rare charm decays from CLEO-c and prospect at BES-III}

With 1.6 million charged $D$ s, the CLEO-c collaboration has searched for the FCNC decays $D^{+} \rightarrow \pi^{+} e^{+} e^{-}$and $D^{+} \rightarrow e^{+} e^{-}$, and the LNV decays $D^{+} \rightarrow \pi^{-} e^{+} e^{+}$and $D^{+} \rightarrow K^{-} e^{+} e^{-}$(chargeconjugate modes are implicit throughout this paper) [7]. For each candidate decay of the form $D^{+} \rightarrow h^{ \pm} e^{\mp} e^{+}$, where $h$ is either $\pi$ or $K$, the energy difference $\Delta E=E_{\text {cand }}-E_{\text {beam }}$ is computed and the beam-constrained mass difference $\Delta m_{b c}=\sqrt{E_{\text {beam }}^{2}-\left|\vec{p}_{c a n d}\right|^{2}}-m_{D^{+}}$, where $E_{\text {cand }}$ and $\vec{p}_{\text {cand }}$ are the measured energy and momentum of the $h^{ \pm} e^{\mp} e^{+}$candidate, $E_{\text {beam }}$ is the beam energy, and $m_{D^{+}}$is the mass of the $D^{+}$meson. Events with $D^{+}$candidates satisfying $-30 \mathrm{MeV} \leq \Delta m_{b c}<30$ $\mathrm{MeV}$ and $-100 \mathrm{MeV} \leq \Delta E<100 \mathrm{MeV}$ are selected for further study. Within this region, the signal box is defined as $-5 \mathrm{MeV} \leq \Delta m_{b c}<5 \mathrm{MeV}$ and $-20 \mathrm{MeV} \leq \Delta E<20 \mathrm{MeV}$, corresponding to $\pm 3 \sigma$ in each variable, as determined by MC simulation. The remainder of the candidate sample was used to assess backgrounds. 


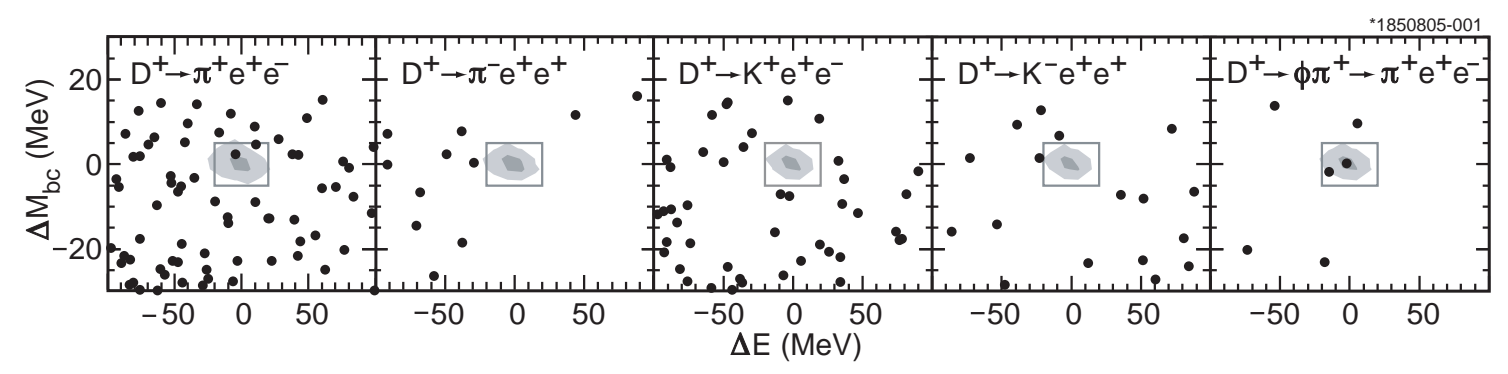

Figure 1: Scatter plots of $\Delta m_{b c}$ vs. $\Delta E$ obtained from data for each decay mode. The signal region, defined by $-20 \mathrm{MeV} \leq \Delta E<20 \mathrm{MeV}$ and $-5 \mathrm{MeV} \leq \Delta m_{b c}<5 \mathrm{MeV}$, is shown as a box. The two contours for each mode enclose regions determined with signal MC simulations to contain $50 \%$ and $85 \%$ of signal events, respectively.

Candidates for the decay of the long-distance decay $D^{+} \rightarrow \pi^{+} \phi \rightarrow \pi^{+} e^{+} e^{-}$are selected using the mass squared of the final-state $e^{+} e^{-}$(equal to the $q^{2}$ of the decay), with $0.9973 \mathrm{GeV}^{2} \leq m_{e^{+} e^{-}}^{2}<$ $1.0813 \mathrm{GeV}^{2}$ defining the $\phi$-resonant region. This region is used both to veto the long-distance $D^{+} \rightarrow \phi \pi^{+} \rightarrow \pi^{+} e^{+} e^{-}$contribution and to measure its branching fraction. Backgrounds in the $D^{+} \rightarrow h^{ \pm} e^{\mp} e^{+}$candidate sample arise from both $D \bar{D}$ and non- $D \bar{D}$ sources. A detailed analysis of backgrounds can be found in Ref. 帆.

CLEO-c results show no evidence for signals, the $90 \%$ confidence level upper limits are set as below:

$$
\begin{aligned}
\mathscr{B}\left(D^{+} \rightarrow \pi^{+} e^{+} e^{-}\right) & <7.4 \times 10^{-6} \\
\mathscr{B}\left(D^{+} \rightarrow \pi^{-} e^{+} e^{+}\right) & <3.6 \times 10^{-6} \\
\mathscr{B}\left(D^{+} \rightarrow K^{+} e^{+} e^{-}\right) & <6.2 \times 10^{-6} \\
\mathscr{B}\left(D^{+} \rightarrow K^{-} e^{+} e^{+}\right) & <4.5 \times 10^{-6}
\end{aligned}
$$

these results for these dielectron modes are significantly more restrictive than previous limits, and reflect sensitivity comparable to the searches for dimuon modes [16]. Due to the dominance of long-distance effects in FCNC modes, they separately measure the branching fraction of the resonant decay $D^{+} \rightarrow \pi^{+} \phi \rightarrow \pi^{+} e^{+} e^{-}$, obtaining $\mathscr{B}\left(D^{+} \rightarrow \phi \pi^{+} \rightarrow \pi^{+} e^{+} e^{-}\right)=\left(2.7_{-1.8}^{+3.6} \pm 0.2\right) \times 10^{-6}$. This is consistent with the product of known world average [16] branching fractions, $\mathscr{B}\left(D^{+} \rightarrow\right.$ $\left.\phi \pi^{+} \rightarrow \pi^{+} e^{+} e^{-}\right)=\mathscr{B}\left(D^{+} \rightarrow \phi \pi^{+}\right) \times \mathscr{B}\left(\phi \rightarrow e^{+} e^{-}\right)=\left[(6.2 \pm 0.6) \times 10^{-3}\right] \times[(2.98 \pm 0.04) \times$ $\left.10^{-4}\right]=(1.9 \pm 0.2) \times 10^{-6}$.

With $20 \mathrm{fb}^{-1}$ data at $\psi(3770)$ peak with the BES-III detector, the sensitivity of the measurements of the rare charm decays are summarized in Tables 2 and 3 for $D^{+}$and $D^{0}$, respectively. For most of them, the sensitivities can be at the order of $10^{-8}$. The current best experimental limits are also listed in the tables 2 and 3 [6].

\section{4. $D^{0}-\bar{D}^{0}$ Mixing, $C P$ Violation at BES-III}

With the design luminosity of $10^{33} \mathrm{~cm}^{-2} \mathrm{~s}^{-1}$, BES-III will have the opportunity to probe for possible new physics which may enter up-type-quark decays. It includes searches for charm mixing, $C P$ violation and rare charm decays. The BES-III charm physics program also includes a variety of measurements that will improve the determination of $\phi_{3} / \gamma$ from $B$-factory experiments. 
Table 2: Current and projected $90 \%$-CL upper limits on rare $D^{+}$decay modes at BES-III with $20 \mathrm{fb}^{-1}$ data at $\psi(3770)$ peak. We assume the selection efficiencies for all modes to be $35 \%$.

\begin{tabular}{llll}
\hline Mode & $\begin{array}{l}\text { Reference } \\
\text { Experiment }\end{array}$ & $\begin{array}{l}\text { Best Upper } \\
\text { limits }\left(10^{-6}\right)\end{array}$ & $\begin{array}{l}\text { BES-III } \\
\left(\times 10^{-8}\right)\end{array}$ \\
\hline$\pi^{+} e^{+} e^{-}$ & CLEO-c [7] & 7.4 & 5.6 \\
$\pi^{+} \mu^{+} \mu^{-}$ & FOCUS [8] & 8.8 & 8.7 \\
$\pi^{+} \mu^{+} e^{-}$ & E791 [9] & 34 & 8.3 \\
$\pi^{-} e^{+} e^{+}$ & CLEO-c [7] & 3.6 & 5.6 \\
$\pi^{-} \mu^{+} \mu^{+}$ & FOCUS [8] & 4.8 & 8.7 \\
$\pi^{-} \mu^{+} e^{+}$ & E791 [9] & 50 & 5.9 \\
$K^{+} e^{+} e^{-}$ & CLEO-c [7] & 6.2 & 6.7 \\
$K^{+} \mu^{+} \mu^{-}$ & FOCUS [8] & 9.2 & 10.5 \\
$K^{+} \mu^{+} e^{-}$ & E791 [9] & 68 & 8.3 \\
$K^{-} e^{+} e^{+}$ & CLEO-c [7] & 4.5 & 6.7 \\
$K^{-} \mu^{+} \mu^{+}$ & FOCUS [8] & 13 & 10.4 \\
$K^{-} \mu^{+} e^{+}$ & E687 [10] & 130 & 8.3 \\
\hline
\end{tabular}

Table 3: Current and projected $90 \%$-CL upper limits on rare $D^{0}$ decay modes at BES-III with $20 \mathrm{fb}^{-1}$ data at $\psi(3770)$ peak.

\begin{tabular}{llll}
\hline Mode & $\begin{array}{l}\text { Reference } \\
\text { Experiment }\end{array}$ & $\begin{array}{l}\text { Best Upper } \\
\text { limits }\left(10^{-6}\right)\end{array}$ & $\begin{array}{l}\text { BES-III } \\
\left(\times 10^{-8}\right)\end{array}$ \\
\hline$\gamma \gamma$ & CLEO [12] & 28 & 5.0 \\
$\mu^{+} \mu^{-}$ & D0 [14] & 2.4 & 17 \\
$\mu^{+} e^{-}$ & E791 [9] & 8.1 & 4.3 \\
$e^{+} e^{-}$ & E791 [9] & 6.2 & 2.4 \\
$\pi^{0} \mu^{+} \mu^{-}$ & E653 [15] & 180 & 12.3 \\
$\pi^{0} \mu^{+} e^{+}$ & CLEO [13] & 86 & 9.7 \\
$\pi^{0} e^{+} e^{-}$ & CLEO [13] & 45 & 7.9 \\
$K_{S} \mu^{+} \mu^{-}$ & E653 [15] & 260 & 10.6 \\
$K_{S} \mu^{+} e^{-}$ & CLEO [13] & 100 & 9.6 \\
$K_{S} e^{+} e^{-}$ & CLEO [13] & 110 & 7.5 \\
$\eta \mu^{+} \mu^{-}$ & CLEO [13] & 530 & 10 \\
$\eta \mu^{+} e^{-}$ & CLEO [13] & 100 & 10 \\
$\eta e^{+} e^{-}$ & CLEO [13] & 110 & 10 \\
\hline
\end{tabular}


The total number of charm mesons accumulated at BES-III will be much smaller than that at the $B$-factories which are about 500 million $D \bar{D}$ pairs for each of them. However, the quantum correlations in the $\psi(3770) \rightarrow D \bar{D}$ system will provide a unique laboratory in which to study charm [17].

\section{1 $D^{0}-\bar{D}^{0}$ Mixing}

$D^{0}-\bar{D}^{0}$ mixing within the SM are highly suppressed due to GIM mechanism, thus, at BES-III, searches for neutral charm mixing and $C P$ violation in the charm decays may be essential to search for some intriguing signals due to new physics.

The time evolution of $D^{0}-\bar{D}^{0}$ system, assuming no $C P$ violation in mixing, is governed by four parameters: $x=\Delta m / \Gamma$ and $y=\Delta \Gamma / 2 \Gamma$ which are the mass and width differences of $D$ meson mass eigenstates and characterize the mixing matrix, $\delta$ the relative strong phase between the Cabibbo favourite (CF) and the doubly-Cabibbo suppressed (DCS) amplitudes and $R_{D}$ the DCS decay rate relative to the CF decay rate. The mixing rate $R_{M}$ is defined as $\frac{1}{2}\left(x^{2}+y^{2}\right)$. The SM based predictions for $x$ and $y$, as well as a variety of non-SM expectations, span several orders of magnitude [1, 18, 19] which is $x \sim y \sim 10^{-3}$. Presently, experimental information about charm mixing parameters $x$ and $y$ comes from the time-dependent analyses.

At the B factories, the wrong-sign (WS) process, $D \rightarrow K^{+} \pi^{-}$, is used to extract the $D^{0}$ mixing parameters by fitting the time-dependent decay rates. The WS process can proceed either through direct doubly-Cabibbo-suppressed (DCS) decay or through mixing followed by the right-sign (RS) Cabibbo favored (CF) decay $D^{0} \rightarrow \bar{D}^{0} \rightarrow K^{+} \pi^{-}$. The two decays can be distinguished by the decay-time distribution. For $|x|,|y| \ll 1$, and assuming negligible $C P V$, the decay-time distribution for $D^{0} \rightarrow K^{+} \pi^{-}$can be expressed as

$$
R_{W S}(t)=e^{-\Gamma t}\left(R_{D}+\sqrt{R_{D}} y^{\prime}(\Gamma t)+\frac{x^{\prime 2}+y^{\prime 2}}{2}(\Gamma t)^{2}\right),
$$

where $x^{\prime}=x \cos \delta+y \sin \delta$ and $y^{\prime}=-x \sin \delta+y \cos \delta$, and $\delta$ is the strong phase between the DCS and CF amplitudes. Recently, a time-dependent analysis in $D \rightarrow K \pi$ has been performed based on 384 $\mathrm{fb}^{-1}$ luminosity at $\Upsilon(4 S)$ by BaBar experiment [20]. By assuming $C P$ conservation, they obtained the following neutral $D$ mixing results

$$
\begin{aligned}
R_{D} & =(3.03 \pm 0.16 \pm 0.10) \times 10^{-3} \\
x^{\prime 2} & =(-0.22 \pm 0.30 \pm 0.21) \times 10^{-3}, \\
y^{\prime} & =(9.7 \pm 4.4 \pm 3.1) \times 10^{-3}
\end{aligned}
$$

The result is inconsistent with the no-mixing hypothesis with a significance of 3.9 standard deviations. The results from $\mathrm{BaBar}$ and Belle are in agreement within 2 standard deviation on the exact analysis of $y^{\prime}$ measurement by using $D \rightarrow K \pi$ as listed in Table 4 .

At $\psi(3770)$ peak, to extract the mixing parameter $y$, one can make use of rates for exclusive $D^{0} \bar{D}^{0}$ combination, where both the $D^{0}$ final states are specified (known as double tags or DT), as well as inclusive rates, where either the $D^{0}$ or $\bar{D}^{0}$ is identified and the other $D^{0}$ decays generically (known as single tags or ST) [17]. With the DT tag technique [24, 25], one can fully consider the quantum correlation in $C=-1$ and $C=+1 D^{0} \bar{D}^{0}$ pairs produced in the reaction $e^{+} e^{-} \rightarrow$ $D^{0} \bar{D}^{0}\left(n \pi^{0}\right)$ and $e^{+} e^{-} \rightarrow D^{0} \bar{D}^{0} \gamma\left(n \pi^{0}\right)$ [26, 27, 17], respectively. 
Table 4: Experimental results used in the paper. Only one error is quoted, we have combined in quadrature statistical and systematic contributions.

\begin{tabular}{c|c|c|c}
\hline \hline Parameter & BaBar $\left(\times 10^{-3}\right)$ & Belle $\left(\times 10^{-3}\right)$ & Technique \\
\hline$x^{\prime 2}$ & $-0.22 \pm 0.37$ [20] & $0.18_{-0.23}^{+0.21}$ [22] & $K \pi$ \\
$y^{\prime}$ & $9.7 \pm 5.4$ [20] & $0.6_{-3.9}^{+4.0}$ [22] & $K \pi$ \\
$R_{D}$ & $3.03 \pm 0.19$ [20] & $3.64 \pm 0.17$ [22] & $K \pi$ \\
$y_{C P}$ & - & $13.1 \pm 4.1$ [2]] & $K^{+} K^{-}, \pi^{+} \pi^{-}$ \\
$x$ & - & $8.0 \pm 3.4$ [23] & $K_{S} \pi^{+} \pi^{-}$ \\
$y$ & - & $3.3 \pm 2.8[23]$ & $K_{S} \pi^{+} \pi^{-}$ \\
\hline \hline
\end{tabular}

For the ST, in the limit of $C P$ conservation, the rate of $D^{0}$ decays into a $C P$ eigenstate is given as [17]:

$$
\Gamma_{f_{\eta}} \equiv \Gamma\left(D^{0} \rightarrow f_{\eta}\right)=2 A_{f_{\eta}}^{2}[1-\eta y]
$$

where $f_{\eta}$ is a $C P$ eigenstate with eigenvalue $\eta= \pm 1$, and $A_{f_{\eta}}=\left|\left\langle f_{\eta}|\mathscr{H}| D^{0}\right\rangle\right|$ is the real-valued decay amplitude.

For the DT case, Gronau et. al. [28] and Xing [29] have considered time-integrated decays into correlated pairs of states, including the effects of non-zero final state phase difference. As discussed in Ref. [28], the rate of $\left(D^{0} \bar{D}^{0}\right)^{C=-1} \rightarrow\left(l^{ \pm} X\right)\left(f_{\eta}\right)$ is described as [28]:

$$
\begin{aligned}
\Gamma_{l ; f_{\eta}} \equiv \Gamma\left[\left(l^{ \pm} X\right)\left(f_{\eta}\right)\right] & =A_{l^{ \pm} X}^{2} A_{f_{\eta}}^{2}\left(1+y^{2}\right) \\
& \approx A_{l^{ \pm} X}^{2} A_{f_{\eta}}^{2},
\end{aligned}
$$

where $A_{l^{ \pm} X}=\left|\left\langle l^{ \pm} X|\mathscr{H}| D^{0}\right\rangle\right|$ is real-valued amplitude for semileptonic decays, here, we neglect $y^{2}$ term since $y \ll 1$.

For $C=-1$ initial $D^{0} \bar{D}^{0}$ state, $y$ can be expressed in term of the ratios of DT rates and the double ratios of ST rates to DT rates [17]:

$$
y=\frac{1}{4}\left(\frac{\Gamma_{l ; f_{+}} \Gamma_{f_{-}}}{\Gamma_{l ; f_{-}} \Gamma_{f_{+}}}-\frac{\Gamma_{l ; f_{-}} \Gamma_{f_{+}}}{\Gamma_{l ; f_{+}} \Gamma_{f_{-}}}\right) .
$$

For a small $y$, its error, $\Delta(y)$, is approximately $1 / \sqrt{N_{l^{ \pm} X}}$, where $N_{l^{ \pm} X}$ is the total number of $\left(l^{ \pm} X\right)$ events tagged with $C P$-even and $C P$-odd eigenstates. The number $N_{l^{ \pm} X}$ of $C P$ tagged events is related to the total number of $D^{0} \bar{D}^{0}$ pairs $N\left(D^{0} \bar{D}^{0}\right)$ through $N_{l^{ \pm} X} \approx N\left(D^{0} \bar{D}^{0}\right)\left[\mathscr{B}\left(D^{0} \rightarrow l^{ \pm}+X\right) \times\right.$ $\left.\mathscr{B}\left(D^{0} \rightarrow f_{ \pm}\right) \times \varepsilon_{\text {tag }}\right] \approx 1.5 \times 10^{-3} N\left(D^{0} \bar{D}^{0}\right)$, here we take the branching ratio-times-efficiency factor $\left(\mathscr{B}\left(D^{0} \rightarrow f_{ \pm}\right) \times \varepsilon_{\text {tag }}\right)$ for tagging $C P$ eigenstates to be about $1.1 \%$ (the total branching ratio into $C P$ eigenstates is larger than about $5 \%$ [16]). We find

$$
\Delta(y)=\frac{ \pm 26}{\sqrt{N\left(D^{0} \bar{D}^{0}\right)}}= \pm 0.003 .
$$

If we take the central value of $y$ from the measurement of $y_{C P}$ at Belle experiment [21], at BES-III experiment, with $20 \mathrm{fb}^{-1}$ data at $\psi(3770)$ peak, the significance of the measurement of $y$ could be around $4.3 \sigma$ deviation from zero [30]. 
We can also take advantage of the coherence of the $D^{0}$ mesons produced at the $\psi(3770)$ peak to extract the strong phase difference $\delta$ between DCS and CF decay amplitudes. Because the $C P$ properties of the final states produced in the decay of the $\psi(3770)$ are anti-correlated [26, 27], one $D^{0}$ state decaying into a final state with definite $C P$ properties immediately identifies or tags the $C P$ properties of the other side. As discussed in Ref. [28], the process of one $D^{0}$ decaying to $K^{-} \pi^{+}$, while the other $D^{0}$ decaying to a $C P$ eigenstate $f_{\eta}$ can be described as

$$
\begin{aligned}
\Gamma_{K \pi ; f_{\eta}} \equiv \Gamma\left[\left(K^{-} \pi^{+}\right)\left(f_{\eta}\right)\right] & \approx A^{2} A_{f_{\eta}}^{2}\left|1+\eta \sqrt{R_{D}} e^{-i \delta}\right|^{2} \\
& \approx A^{2} A_{f_{\eta}}^{2}\left(1+2 \eta \sqrt{R_{D}} \cos \delta\right),
\end{aligned}
$$

where $A=\left|\left\langle K^{-} \pi^{+}|\mathscr{H}| D^{0}\right\rangle\right|$ and $A_{f_{\eta}}=\left|\left\langle f_{\eta}|\mathscr{H}| D^{0}\right\rangle\right|$ are the real-valued decay amplitudes, and we have neglected the $y^{2}$ terms in Eq. (4.7). In order to estimate the total sample of events needed to perform a useful measurement of $\delta$, one define [28, 1] an asymmetry

$$
\mathscr{A} \equiv \frac{\Gamma_{K \pi ; f_{+}}-\Gamma_{K \pi ; f_{-}}}{\Gamma_{K \pi ; f_{+}}+\Gamma_{K \pi ; f_{-}}}
$$

where $\Gamma_{K \pi ; f_{ \pm}}$is defined in Eq. (4.7), which is the rates for the $\psi(3770) \rightarrow D^{0} \bar{D}^{0}$ configuration to decay into flavor eigenstates and a $C P$-eigenstates $f_{ \pm}$. Eq. (4.7) implies a small asymmetry, $\mathscr{A}=2 \sqrt{R_{D}} \cos \delta$. For a small asymmetry, a general result is that its error $\Delta \mathscr{A}$ is approximately $1 / \sqrt{N_{K^{-}} \pi^{+}}$, where $N_{K^{-}} \pi^{+}$is the total number of events tagged with $C P$-even and $C P$-odd eigenstates. Thus one obtain

$$
\Delta(\cos \delta) \approx \frac{1}{2 \sqrt{R_{D}} \sqrt{N_{K^{-}} \pi^{+}}} .
$$

The expected number $N_{K^{-} \pi^{+}}$of $C P$-tagged events can be connected to the total number of $D^{0} \bar{D}^{0}$ pairs $N\left(D^{0} \bar{D}^{0}\right)$ through [28]

$$
N_{K^{-} \pi^{+}} \approx N\left(D^{0} \bar{D}^{0}\right) \mathscr{B}\left(D^{0} \rightarrow K^{-} \pi^{+}\right) \times \mathscr{B}\left(D^{0} \rightarrow f_{ \pm}\right) \times \varepsilon_{\text {tag }} \approx 4.2 \times 10^{-4} N\left(D^{0} \bar{D}^{0}\right)
$$

here, as in Ref [28], we take the branching ratio-times-efficiency factor $\mathscr{B}\left(D^{0} \rightarrow f_{ \pm}\right) \times \varepsilon_{\text {tag }}=1.1 \%$. With the measured $R_{D}=(3.03 \pm 0.19) \times 10^{-3}$ and $\mathscr{B}\left(D^{0} \rightarrow K^{-} \pi^{+}\right)=3.8 \%$ [16, one found [28]

$$
\Delta(\cos \delta) \approx \frac{ \pm 444}{\sqrt{N\left(D^{0} \bar{D}^{0}\right)}} .
$$

At BES-III, about $72 \times 10^{6} D^{0} \bar{D}^{0}$ pairs can be collected with 4 years of running. Considering both $K^{-} \pi^{+}$and $K^{+} \pi^{-}$final states, we thus estimate that one may be able to reach an accuracy of about 0.04 on $\cos \delta$.

At BES-III, the measurement of $R_{M}$ can be performed unambiguously with the following reactions [26]:

$$
\begin{aligned}
& \text { (i) } e^{+} e^{-} \rightarrow \psi(3770) \rightarrow D^{0} \bar{D}^{0} \rightarrow\left(K^{ \pm} \pi^{\mp}\right)\left(K^{ \pm} \pi^{\mp}\right), \\
& \text { (ii) } e^{+} e^{-} \rightarrow \psi(3770) \rightarrow D^{0} \bar{D}^{0} \rightarrow\left(K^{-} e^{+} v\right)\left(K^{-} e^{+} v\right), \\
& \text { (iii) } e^{+} e^{-} \rightarrow D^{-} D^{*+} \rightarrow\left(K^{+} \pi^{-} \pi^{-}\right)\left(\pi_{\text {soft }}^{+}\left[K^{+} e^{-} v\right]\right) .
\end{aligned}
$$


Reaction $(i)$ in Eq. (4.11) can be normalized to $D^{0} \bar{D}^{0} \rightarrow\left(K^{-} \pi^{+}\right)\left(K^{+} \pi^{-}\right)$, the following timeintegrated ratio is obtained by neglecting $C P$ violation:

$$
\frac{N\left[\left(K^{-} \pi^{+}\right)\left(K^{-} \pi^{+}\right)\right]}{N\left[\left(K^{-} \pi^{+}\right)\left(K^{+} \pi^{-}\right)\right]} \approx \frac{x^{2}+y^{2}}{2} \equiv R_{M} .
$$

For the case of semileptonic decay, as (ii) in Eq. (4.11), we have

$$
\frac{N\left(l^{ \pm} l^{ \pm}\right)}{N\left(l^{ \pm} l^{\mp}\right)}=\frac{x^{2}+y^{2}}{2} \equiv R_{M}
$$

In the limit of $C P$ conservation, by combing the measurements of $x$ in $D^{0} \rightarrow K_{S} \pi \pi$ and $y_{C P}$ from Belle, one obtain $R_{M}=(1.18 \pm 0.6) \times 10^{-4}$. With $20 \mathrm{fb}^{-1}$ data at BES-III, about 12 events for the precess $D^{0} \bar{D}^{0} \rightarrow\left(K^{ \pm} \pi^{\mp}\right)\left(K^{ \pm} \pi^{\mp}\right)$ can be produced. One can observe 3.0 events after considering the selection efficiency at BES-III, which could be about $25 \%$ for the four charged particles. The background contamination due to double particle misidentification is about 0.6 event with 20 $\mathrm{fb}^{-1}$ data at BES-III [31].

\section{2 $C P$ Violation in $D$ System}

For the direct $C P$ violation, the SM predictions are as large as $0.1 \%$ for $D^{0}$ decays, and $1 \%$ level for $D^{+}$and $D_{S}$ decays [32]. At BES-III, one can also look at $C P$ violation by exploiting the quantum coherence at the $\psi(3770)$. Consider the case where both the $D^{0}$ and the $\bar{D}^{0}$ decay into $C P$ eigenstates, then the decays $\psi(3770) \rightarrow f_{+}^{i} f_{+}^{i}$ or $f_{-}^{i} f_{-}^{i}$ are forbidden, where $f_{+}\left(f_{-}\right)$denotes a $C P+$ eigenstate $\left(C P\right.$ - eigenstate). This is because $C P\left(f_{ \pm}^{i} f_{ \pm}^{i}\right)=C P\left(f_{ \pm}^{i}\right) C P\left(f_{ \pm}^{i}\right)(-1)^{l}=-1$, while, for the $l=1 \psi(3770)$ state, $C P(\psi(3770))=+1$. Thus the observation of a final state such as $\left(K^{+} K^{-}\right)\left(\pi^{+} \pi^{-}\right)$constitutes evidence of $C P$ violation. For $\left(K^{+} K^{-}\right)\left(\pi^{+} \pi^{-}\right)$mode, the sensitivity at BES-III is about $1 \%$ level. Moreover, all pairs of the $C P$ eigenstates, where both eigenstates are even or both are odd, can be summed over for the $C P$ violation measurements at BES-III.

\section{Summary}

In summary, the rare charm decays are searched for at CLEO-c. No evidence is found either for the rare (FCNC) decays or for the forbidden (LNV) decays of charged $D$ mesons to three-body final states with dielectrons. The sensitivities of the rare charm decays are presented by assuming $20 \mathrm{fb}^{-1}$ at $\psi(3770)$ peak with BES-III detector. The measurements at BES-III for these rare decays can be at the level of $10^{-8}$. The sensitivities for the neutral $D$ mixing and $C P$ violation are also studied, we find that sensitivity for the lifetime difference $y$ can be 0.003 with $20 \mathrm{fb}^{-1}$ data at $\psi(3770)$ peak, and the significance will be $4.3 \sigma$ if $y$ is at $1 \%$ level.

\section{References}

[1] G. Burdman and I. Shipsey, Ann. Rev. Nucl. Part. Sci. 53, 431 (2003).

[2] G. Burdman, E. Golowich, J. L. Hewett, S. Pakvasa, Phys. Rev. D66, 014009 (2002).

[3] S. Fajfer, S. Prelovsek and P. Singer, Phys. Rev. D 64, 114009 (2001). 
[4] I. Shipsey, hep-ex/0607070.

[5] Y. .F. Wang, Int. J. Mod. Phys. A21, 5371 (2006).

[6] H. Li, Nucl. Phys. Proc. Suppl. 162,312 (2006).

[7] Q. He et al., (CLEO-c Collaboration), Phys. Rev. Lett. 95 , 221802 (2005).

[8] J. M. Link et al., Phys. Lett. B572 (2003) 21.

[9] E. M. Aitala et al., Phys. Lett. B462 (1999) 401.

[10] P. L. Frabetti et al., Phys. Lett.B398 (1997) 239.

[11] G. Burdman and I.Shipsey, Ann. Rev. Nucl. Part. Sci. 53 (2003) 431; S. Bianco, F. L. Fabbri,

D. Benson and I. Bigi, Riv. Nuovo Cim. 26N7 (2003) 1.

[12] T. E. Coan et al., Phys. Rev. Lett. 90 (2003) 101801.

[13] A. Freyberger et al., Phys. Rev. Lett. 76 (1996) 3065; erratum, Phys. Rev. Lett. 77 (1996) 2147.

[14] A. Korn et al., hep-ex/0305054.

[15] K. Kodama et al., Phys. Lett. B345 (1995) 85.

[16] W. M. Yao et. al., (Partcle Data Group), J. Phys.G 33, 1(2006).

[17] D. M. Asner and W. M. Sun Phys. Rev. D73, 034024 (2006);D. M. Asner et. al., Int. J. Mod. Phys. A21, 5456 (2006); W. M. Sun, hep-ex/0603031, AIP Conf. Proc. 842:693-695 (2006).

[18] A. F. Falk, Y. Nir, and A. Petrov, JHEP12, 019 (1999).

[19] D. Atwood, A. A. Petrov, Phys. Rev. D71, 054032 (2005).

[20] B. Aubert, et. al., (BaBar Collaboration), Phys. Rev. Lett. 98, 211802 (2007).

[21] M. Staric et. al., (Belle Collaboration), Phys. Rev. Lett. 98,211803 (2007).

[22] L. M. Zhang et. al., (Belle Collaboration), Phys. Rev. Lett. 96, 151801 (2006).

[23] L. M. Zhang et al., (Belle Collaboration) arXiv:hep-ex/0704.1000.

[24] R. M. Baltrusaitis, et. al., (MARK III Collaboration), Phys. Rev. Lett. 56, 2140(1986).

[25] J. Adler, et. al., (MARK III Collaboration), Phys. Rev. Lett. 60, 89 (1988).

[26] I. I. Bigi, Proceed. of the Tau-Charm Workshop, L. V. Beers (ed.), SLAC-Report-343, page 169, (1989).

[27] I. Bigi, A. Sanda, Phys. Lett. B171, 320(1986).

[28] M. Gronau, Y. Grossman, J. L. Rosner, Phys. Lett. B508, 37 (2001).

[29] Z. Z. Xing, Phys. Rev. D55, 196(1997);

Z. Z. Xing, Phys. Lett. B372,317(1996).

[30] X. D. Cheng, K. L. He, H. B. Li, Y. F. Wang, M. Z. Yang, Phys. Rev. D75, 094019 (2007).

[31] Y. Z. Sun et al., High Energy Phys. Nucl. Phys. 31, 423(2007).

[32] F. Buccella et al., Phys. Lett. B379 (1996) 249. 